\title{
Gérard Dussouy
}

Maître de conférences à l'Université Montesquieu - Bordeaux IV. (2002)

professeur agrégé de géographie, chercheur au Centre d'analyse politique comparée de l'Université Montesquieu de Bordeaux

(2002)

\section{"Vers une géopolitique systémique."}

Un document produit en version numérique par Jean-Marie Tremblay, bénévole, professeur de sociologie retraité du Cégep de Chicoutimi

Courriel: jean-marie_tremblay@uqac.ca

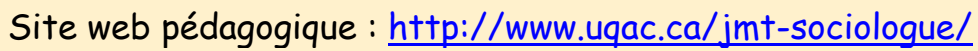

Dans le cadre de: "Les classiques des sciences sociales" Une bibliothèque numérique fondée et dirigée par Jean-Marie Tremblay, professeur de sociologie au Cégep de Chicoutimi

Site web: http://classiques.uqac.cal

Une collection développée en collaboration avec la Bibliothèque

Paul-Émile-Boulet de l'Université du Québec à Chicoutimi

Site web: $h+t p: / /$ bibliotheque.uqac.cal 


\section{Politique d'utilisation de la bibliothèque des Classiques}

Toute reproduction et rediffusion de nos fichiers est interdite, même avec la mention de leur provenance, sans l'autorisation formelle, écrite, du fondateur des Classiques des sciences sociales, Jean-Marie Tremblay, sociologue.

Les fichiers des Classiques des sciences sociales ne peuvent sans autorisation formelle:

- être hébergés (en fichier ou page web, en totalité ou en partie) sur un serveur autre que celui des Classiques.

- servir de base de travail à un autre fichier modifié ensuite par tout autre moyen (couleur, police, mise en page, extraits, support, etc...),

Les fichiers (.html, .doc, .pdf, .rtf, .jpg, .gif) disponibles sur le site Les Classiques des sciences sociales sont la propriété des Classiques des sciences sociales, un organisme à but non lucratif composé exclusivement de bénévoles.

Ils sont disponibles pour une utilisation intellectuelle et personnelle et, en aucun cas, commerciale. Toute utilisation à des fins commerciales des fichiers sur ce site est strictement interdite et toute rediffusion est également strictement interdite.

L'accès à notre travail est libre et gratuit à tous les utilisateurs. C'est notre mission.

Jean-Marie Tremblay, sociologue

Fondateur et Président-directeur général, LES CLASSIQUES DES SCIENCES SOCIALES. 
Cette édition électronique a été réalisée par Jean-Marie Tremblay, bénévole, professeur de sociologie au Cégep de Chicoutimi à partir de :

\section{Gérard Dussouy}

professeur agrégé de géographie, chercheur au Centre d'analyse politique comparée de l'Université Montesquieu de Bordeaux

\section{"Vers une géopolitique systémique".}

Un article publié dans la Revue internationale et stratégique, no 47, mars 2002, pp. 53-66. Paris : Les Éditions Armand Colin.

Courriel : gerard.dussouy@wanadoo.fr

Polices de caractères utilisée:

Pour le texte: Comic Sans, 12 points.

Pour les notes de bas de page : Comic Sans, 10 points.

Édition électronique réalisée avec le traitement de textes Microsoft Word 2008 pour Macintosh.

Mise en page sur papier format : LETTRE US, $8.5^{\prime \prime} \times 11^{\prime \prime}$

Édition numérique réalisée le 25 août 2011 à Chicoutimi, Ville de Saguenay, Québec. 


\section{Gérard Dussouy}

professeur agrégé de géographie, chercheur au Centre d'analyse politique comparée de l'Université Montesquieu de Bordeaux

\section{"Vers une géopolitique systémique".}

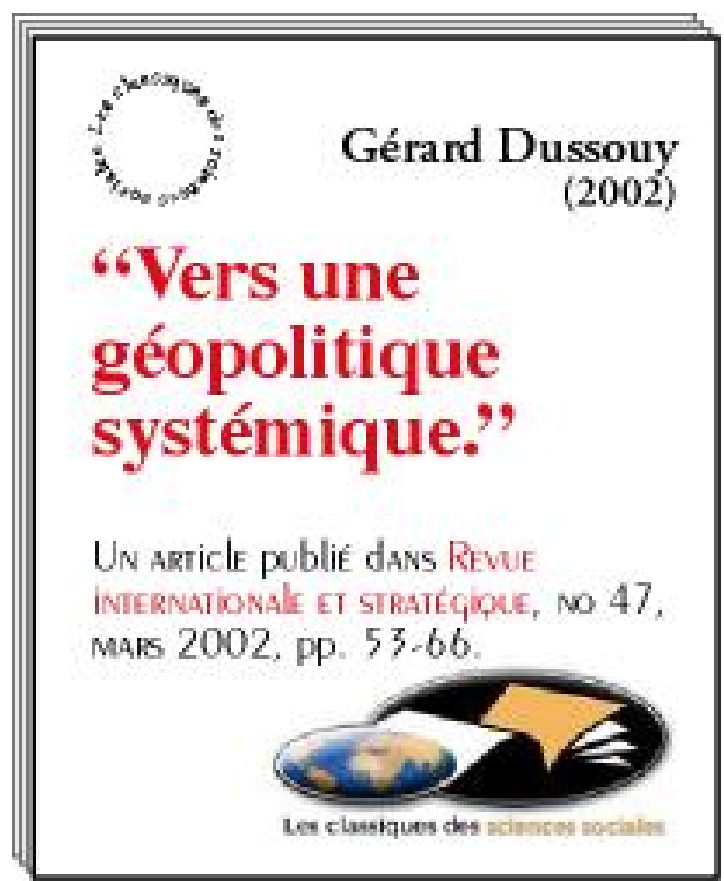

Un article publié dans la Revue internationale et stratégique, no 47, mars 2002, pp. 53-66. Paris : Les Éditions Armand Colin. 


\section{Table des matières}

Introduction

La géopolitique comme représentation cognitive de l'espace mondial

Subjectivité des points de vue et relativité des modèles

Jeux d'acteurs et "respatialisation des concepts"

L'axe Washington-Moscou-New Delhi: "géopolitique heuristique » ou réalité?

Critique géoéconomique de l'homogénéité du modèle capitaliste mondial

L'hétérogénéité spatiale et temporelle de l'économie mondiale Une régulation par zones de l'économie mondiale?

Perspectives géoculturelles

Les cultures sont-elles solubles dans la mondialisation?

Renouvellement mimétique de paradigmes anciens

Le "nouveau multilatéralisme »: un dialogue intercivilisationnel

Géopolitique/géostratégie: couple référentiel d'une même analyse spatio-politique 
[53]

\section{Gérard Dussouy \\ professeur agrégé de géographie, chercheur \\ au Centre d'analyse politique comparée \\ de l'Université Montesquieu de Bordeaux \\ "Vers une géopolitique systémique."}

Un article publié dans la Revue internationale et stratégique, no 47, mars 2002, pp. 53-66. Paris : Les Éditions Armand Colin.

\section{Introduction}

\section{Retour à la table des matières}

Quand, voilà quelques années, le terme de « géopolitique » réapparut dans la littérature traitant des problèmes internationaux, et celui de "géostratégie», à la fois moins usité et moins connoté, se diffusait parmi les experts des relations Est-Ouest alors largement dominées par les rapports militaires, le général Lucien Poirier écrivit: "L'espace est l'une des catégories usuelles de la pensée stratégique, laquelle s'inscrit dans la dimension "géo". Dire géostratégie est tautologique. » 1 L'art de la guerre consistant, dans une large mesure, à démontrer une compétence aiguë dans l'utilisation du terrain, la conviction de l'officier supérieur français quant à l'intégration du concept d'espace à la stratégie militaire n'est pas à mettre en doute. Pourtant, à cette époque où le rôle écrasant des arsenaux balistico-nucléaires contribuait à homogénéiser le champ de la réflexion militaire, des auteurs tels que Colin S. Gray insistaient sur la nécessité de toujours

1 Général Lucien Poirier, in la postface du livre du général Jean Colin, Les transformations de la guerre, Paris, Economica, 1989, p. 268. 
garder à l'esprit les hétérogénéités de la géographie, du monde réel 2 . Soit, en l'occurrence, la répartition des terres et des mers qui exigeait que l'on développât une pensée géostratégique explicite, même à l'âge du nucléaire. Il s'agissait bien sûr, par cette démarche, d'intégrer toutes les disjonctions spatiales et toute la variété des situations locales qui posaient déjà problème à la théorie de la dissuasion, et qui ont pris l'ampleur que l'on constate tous les jours depuis la fin de la guerre froide, depuis que les états-majors n'envisagent plus que des conflits locaux. Le général L. Poirier a lui-même, avec beaucoup de lucidité, tiré toutes les conséquences de ce changement, posant que désormais « la géopolitique-géostratégie se donne pour objet de décrire et d'expliquer les relations interactives de chacun des acteurs avec chacun et avec tous, par les corrélations entre deux critères d'évaluation: leurs sites et attributs géographiques, et leurs facteurs de puissance et de vulnérabilité [...] ». Elle permet enfin de "se représenter les états successifs de la carte du monde par les variations conjuguées, dans l'espace planétaire, des positions et postures relatives des acteurs pris deux à deux $\gg 3$.

Plus récemment, aux termes de "géopolitique » et de "géostratégie », ont été ajoutés les substantifs « géoéconomie » et "géoculture». On entend aussi parfois "géohistoire» et, beaucoup plus fréquemment, les adjectifs « géoéconomique » et "géoculturel». Or la généralisation de ces termes est concomitante de la multiplication, dans tous les champs des sciences politiques et sociales, des analyses du phénomène [54] de la globalisation, qui appellent à la prise en considération des hétérogénéités de l'espace mondial. Ici, c'est la théorie de la modernisation qui est en cause. En effet, les Modernes ont privilégié le temps et ont dévalué l'espace parce qu'ils étaient convaincus de l'homogénéisation inéluctable de ce dernier et, par conséquent, de son indifférence à l'évolution du monde. Il leur suffisait d'attendre les accomplissements du progrès humain qui rendait la « spatialité silencieu-

2 Colin S. Gray, The Geopolitics of the Nuclear Era. Heartland, Rimlands and the Technological Revolution, New York, Crane \& Russac, 1977.

3 Général Lucien Poirier, La crise des fondements, Paris, Economica/ISC, 1994, p. 170. 
se », selon la formule avisée d'Edward W. Soja ${ }^{4}$. Mais, pas plus que le temps, l'espace n'est figé. Au contraire, la fragmentation du social, du culturel et du politique, qui, sous couvert d'homogénéisation économique, est la caractéristique essentielle de la postmodernité 5 , va de pair avec des recompositions spatiales ou des reterritorialisations, infra- et supranationales, déterminées par des stratégies d'acteurs d'origines et de natures très diverses.

En somme, la redondance du préfixe "géo », d'une discipline à une autre, s'explique principalement par les deux raisons connexes suivantes: - La prégnance ou le retour, selon les cas, de la variable spatiale dans les sciences militaires, politiques, économiques et sociales, qui réhabilitent le contextuel aux dépens des théories trop réductrices de la dissuasion et de la modernisation. L'une et l'autre des deux hypothèses s'expliquent à la fois par l'unification du monde et par la rencontre du global et du local, bien que la première ("la prégnance ») s'articule de préférence sur des permanences, tandis que la seconde (« le retour ») a pour cause l'extension et la différenciation des espaces stratégiques investis par les différents acteurs, quels qu'ils soient, et ce, suite au développement considérable des technologies de communication. Peu entendu, Michel Foucault avait déjà insisté sur la nécessaire prise en compte de la richesse du rapport multiple à l'espace, des positions et des relations spatiales, parlant, à cet égard, d'hétérotopies 6 .

- L'émergence ou la radicalisation de la tension entre homogénéisation et hétérogénéisation dans les processus de globalisation économique et culturelle. La théorisation de cette tension nous est apparue ${ }^{7}$, ainsi qu'à d'autres auteurs aussi différents que Roland Robert-

4 Edward W. Soja, Postmodern Geographies. The Reassertion of Space in Critical Social Theory, New York/Londres, Verso, 1989, p. 13.

5 Michel Maffésoli, L'instant éternel. Le retour du tragique dans les sociétés postmodernes, Paris, Denoël, 2000. Pour ne citer que cet ouvrage.

6 Michel Foucault, «Espace, savoir et pouvoir», repris in Michel Foucault, Dits et Écrits, Paris, Gallimard, 1994, t. 2.

7 Gérard Dussouy, Quelle géopolitique au XXIe siècle?, Bruxelles, Complexe, 2001. 
son 8 et Arjun Appadurai ${ }^{9}$, comme le nouveau paradigme qui pourrait permettre d'approcher au mieux la réalité des relations internationales, tant il est vrai que les flux sont à la fois unificateurs et disjonctifs, qu'ils fissurent les frontières en même temps que les localismes s'affirment ou reviennent en force, et que le transnational déstabilise les États qui recherchent alors des solutions collectives de reterritorialisation. Toutefois, un tel paradigme ne saurait être limité à la recherche de couples de contraires, car l'approche dialectique est trop réductrice et tend à exagérer l'importance des conflits. C'est pourquoi, de notre côté, nous avons mis en oeuvre une «trialectique » qui dépasse le domaine polaire, parce qu'à la confrontation ou à la supplantation - qui représente l'alternative dialectique classique -, elle ajoute la possibilité d'une coexistence des opposés, d'une coprésence des ethnocentrismes.

[55]

C'est donc à partir de ces deux lignes directrices qu'il nous est possible d'apprécier la légitimité et la pertinence des différents termes en vogue, lesquels, d'ailleurs, renvoient les uns aux autres tant ils sont en interaction.

\section{La géopolitique comme représentation cognitive de l'espace mondial}

\section{Retour à la table des matières}

Dès l'origine de leurs oeuvres, le défi que les géopoliticiens se lancèrent à eux-mêmes fut celui d'accéder à une vue panoptique du monde. Avec l'avènement du «One world», la nécessité de saisir le « Tout mondial » dans sa multiplicité n'en est devenue que plus forte. C'est pourquoi, dans sa nouvelle démarche, on peut assimiler la géopolitique à

8 Roland Robertson, «Glocalization: Time-Space and HomogeneityHeterogeneity », in Global Modernities, Londres, Sage, 1995, p. 25-44.

9 Arjun Appadurai, Après le colonialisme. Les conséquences culturelles de la globalisation, Paris, Payot, 2001. 
une ontologie spatiale. Elle est une réflexion sur l'organisation et le devenir de la Terre et des hommes qu'elle porte. En cela réside sa nécessité consubstantielle, mais les interrogations qu'elle suscite n'en demeurent pas moins difficiles à surmonter. Geraoid O'Tuathail a d'ailleurs pris un malin plaisir à relever les déviances, les tropismes et autres défectuosités qui ont affecté les diverses représentations géopolitiques depuis le début du XIXe siècle jusqu'à celle qui prévalut lors du conflit de Bosnie 10.

\section{Subjectivité des points de vue et relativité des modèles}

Toute vision, il est vrai, est subjective et perspectiviste en ce sens qu'elle relève d'un point de vue central et souverain. Aucun observateur ne possédant la connaissance objective et parfaite du monde sur lequel il se penche, se pose le «problème des préjugés » soulevé par le philosophe allemand Hans-Georg Gadamer ${ }^{11}$. Selon lui, le problème se subdivise de la façon suivante. En premier lieu, il se trouve que toute compréhension relève essentiellement du préjugé, soit une pré-opinion qui résiste ou non à l'épreuve des faits. Le philosophe, qui parvient à cette conclusion au terme d'une réflexion sur la compréhension dans les sciences de l'esprit au moyen d'une lecture herméneutique de la phénoménologie husserlienne et de la théorie de l'Histoire, estime que les Lumières ont commis l'erreur de déprécier systématiquement le préjugé, car il en existe qui sont légitimes. En second lieu, tout acteur ou tout observateur est impliqué dans une situation, c'est-à-dire dans un lieu où il se tient et qui limite les possibilités de sa vision. C'est pourquoi le concept d'horizon, lié à celui de situation, apparaît si important à H.-G. Gadamer, l'horizon étant « le champ de vision qui comprend et inclut tout ce que l'on peut voir d'un point précis $» 12$.

\footnotetext{
10 Geraoid O'Tuathail, Critical Geopolitics, Londres, Routledge, 1996.

11 Hans-Georg Gadamer, Vérité et méthode. Les grandes lignes d'une herméneutique philosophique, Paris, Le Seuil, 1976, p. 286-312.

12 Ibid.
} 
Un analyste connu des relations internationales, Robert W. Cox, qui s'est inspiré de la conception gramscienne de l'hégémonie pour justifier de son propre engagement intellectuel, a depuis longtemps convenu de cette double limite: «Une théorie est toujours pour quelqu'un et pour quelque chose. Toutes les théories ont une perspective. Les perspectives dérivent d'une position dans le temps et dans l'espace, particulièrement dans le temps et l'espace politiques et sociaux. Le monde est perçu d'un point de vue définissable en termes de nation ou de classe sociale, de domination ou de subordination, de puissance montante ou déclinante, d'immobilité ou de crise, d'expériences vécues et d'espérances et d'attentes pour le futur. »13 C'est ainsi que [56] l'observateur, à la fois sujet et objet de l'Histoire, soumis aux pressions et aux contraintes de son contexte de vie, ne se débarrasse jamais complètement des préjugés qui lui ont été inculqués. De là, le préjugé ethnocentrique que l'on décèle dans la plupart des représentations géopolitiques classiques, auquel répondit plus tard le préjugé cosmopolite d'essence économiste et humanitariste d'un grand nombre de théories contemporaines. Aujourd'hui, l'antagonisme des deux préjugés, celui qui tient à l'exceptionnalité de chaque culture et celui qui postule l'unité de l'humanité et l'existence d'une culture humaine, tend à focaliser le débat sur le monde, ce qui ne rend pas facile la tâche de ceux qui veulent se tenir à égale distance des deux extrémités. Néanmoins, du fait même de cette controverse et en raison de la diversité des horizons, c'est dans l'intersubjectivité - autrement dit, grâce à la synthèse intersubjective des paradigmes et des modèles que ceux-ci inspirent - que réside la pertinence de la géopolitique.

Il faut bien admettre que, de ce point de vue, l'enjeu est d'une complexité extrême. Les horizons des acteurs de la vie internationale sont brouillés par la globalisation, par les déterritorialisations et les interférences de toutes natures. Les différents champs (diplomaticomilitaire, économique, démographique et symbolique) dans lesquels ceux-là se meuvent, se parasitent mutuellement. Tout événement important ou toute perturbation dans l'un d'entre eux se répercute dans

13 Robert W. Cox, « Social Forces, States and World Order : Beyond International Relations Theory », in Robert O. Keohane, Neorealism and its Critics, New York, Columbia University Press, 1986, p. 204-249. 
les autres, pouvant entraîner des réactions en chaîne le long de l'axe qui relie les trois niveaux macro (supranational et transnational), meso (national) et micro (local, infranational) de l'espace mondial. En outre, ces répercussions ne sont pas de sens univoque, car, loin d'être un univers homogène, le monde est un système de contextes. Dans ces conditions, le panoptisme qui légitime la géopolitique doit se garder de tout essentialisme, de tout point de vue unique et surplombant, pour proposer des configurations alternatives, parce que conçues selon différentes hypothèses d'évolution. Cette démarche est sans aucun doute indispensable parce qu'elle se loge au creux de la théorie des relations internationales qui ne peut plus, elle-même, ignorer la variable spatiale, au titre d'un universalisme idéalisé ou parce que la géographie est réputée avoir été élevée au rang de déterminant en dernier ressort par l'ancienne géopolitique, alors qu'elle servait surtout de support au culturalisme ${ }^{14}$.

\section{Jeux d'acteurs et « respatialisation des concepts »}

\section{Retour à la table des matières}

L'espace est un système de champs ${ }^{15}$, c'est-à-dire d'espaces spécifiques structurés par des acteurs qui privilégient deux formes compatibles : le territoire et le réseau. Le premier est une portion d'espace appropriée, délimitée et close. Le second est un dispositif stratégique de pouvoir dans l'espace, distancié, ouvert, mobile et visible ou invisible ${ }^{16}$. De surcroît, l'espace étant discontinu et hétérogène par constitution, sa prise en compte invite la théorie à demeurer attentive aux faits, et toute représentation, à montrer un minimum de positivité. Avant toute chose, il importe de prendre en considération les straté-

14 Gérard Dussouy, Les représentations géopolitiques classiques du monde, à paraître aux Éditions Complexe.

15 Pierre Bourdieu, «Quelques propriétés du champ», in Pierre Bourdieu, Questions de sociologie, Paris, Éd. de Minuit, 1980, p. 113-120.

16 Réseau et pouvoir, ouvrage collectif du Centre d'analyse politique comparée, Université Montesquieu - Bordeaux IV, à paraître chez L'Harmattan. 
gies des acteurs qui organisent l'espace et lui confèrent sa configuration.

Ce concept de configuration, qui est, selon nous, le fondement du renouvellement de la géopolitique, a rarement été analysé en profondeur. On peut partir, cependant, [57] de la définition qu'en a donnée Norbert Elias: "C'est la figure globale toujours changeante que forment les joueurs; elle inclut non seulement leur intellect, mais toute leur personne, les actions et les relations réciproques. Comme on peut le voir, cette configuration forme un ensemble de tensions. L'interdépendance des joueurs, condition nécessaire à l'existence d'une configuration spécifique, est une interdépendance en tant qu'alliés, mais aussi en tant qu'adversaires. »17 Adaptée à notre objet, elle devient le système spatial global qu'organisent les stratégies des acteurs, en fonction des corrélations de forces, mais aussi des intentions des principaux d'entre eux, guidés par leurs intérêts et leurs mythes sociaux. La configuration offre une certaine plasticité : elle est transformable en même temps qu'elle exerce une forte contrainte. Ainsi, la stratégie se moule à la contingence pour inférer une recontextualisation, car « une configuration doit être issue d'une certaine configuration précédente ou même de toute une série de configurations d'un type bien défini, sans pour autant démontrer que ces premières configurations devraient nécessairement se transformer en celles qui leur succèdent $\gg 18$. C'est dans cette succession de configurations que l'on peut trouver une justification à la notion de géohistoi$r e$, si l'on entend par là la recherche dans le temps d'antécédents spatiaux à une construction ou à une action politiques. Par exemple, la Triade, qui est la configuration caractéristique du système global capitaliste, est l'héritière du système géostratégique qui avait été mis en place par les États-Unis au lendemain de la Seconde Guerre mondiale, par reconstruction à l'identique de l'Europe et du Japon.

L'intégration de l'espace, et de toute la connaissance géographique qui l'emplit, à la théorie, exerce sur celle-ci un « effet de positivi-

17 Norbert Elias, Qu'est ce que la sociologie? Paris, Éd. de l'Aube, 1991.

18 Ibid. 
té »19. Il lui faut décrire, analyser un environnement qui s'impose aux acteurs et qui, justement, «suppose une prise en charge théorique $\gg 20$, parce que l'espace n'est pas isotrope. Au contraire, il est polarisé et déséquilibré, parcouru de fissures. Il est produit et intériorisé par des sociétés à stratification sociale et politique plus ou moins homogène et hétérogène. Grâce à cette positivité, qui se distingue de l'empirie par son aspect impératif, la " géographie heuristique», selon l'expression de Francois Dagognet, que se doit d'être la géopolitique, éclaire l'observateur par «les superpositions (milieux et mouvements) : [et] par les dessins distinctifs qu'elle nous propose $» 21$. Soit, d'une part, par ses emboîtements, interfaces, confluences, lignes de contact, qui sont aussi bien des regroupements d'États, des rencontres culturelles ou civilisationnelles, des expansions de flux ou de réseaux, des failles géohistoriques, des dominances de différentes natures, etc. Soit, d'autre part, par ses démarcations, ses zones centrales et périphériques, ses fragments d'espace, ses diffusions, ses décalages démographiques, etc. Le tout génère un savoir multifactoriel sur les rapports entre l'organisation de l'espace mondial et la vie politique, un savoir qui a un double effet, un « effet de politisation » et un « effet de critique »22. La politisation est inhérente au pouvoir qu'induit toute connaissance, toute anticipation compréhensive des affaires du monde. Pour une large part, l'efficacité stratégique consiste à comprendre un peu plus tôt que l'autre la configuration dans laquelle on opère, et ce qu'il adviendra des facteurs mis en jeu. L'effet de critique est [58] la conséquence du sens du relatif qui occupe l'esprit du géographe. Son exercice revient à effectuer ce que Jocelyn Benoist appelle la « respatialisation des concepts» 23 , c'est-à-dire à recontex-

19 Jocelyn Benoist, «En quoi la géographie peut-elle importer à la philosophie? », in Jocelyn Benoist et Fabio Merlini (sous la dir.), Historicité et spatialité. Le problème de l'espace dans la pensée contemporaine, Paris, Librairie philosophique J. Vrin, 2001, p. 222-247.

20 Ibid., p. 227.

21 Francois Dagognet, Une épistémologie de l'espace concret. Néo-géographie, Paris, Librairie philosophique J. Vrin, 1977, p. 150.

22 J. Benoist, op. cit., p. 241-247.

23 Jocelyn Benoist, «Rompre avec l'idéalisme historique: re-spatialiser nos concepts», in J. Benoist et F. Merlini (sous la dir.), op. cit., p. 99-103. 
tualiser selon les différents lieux, culturels notamment, les concepts universels trop régulièrement absolutisés, tels que la démocratie. La respatialisation permet la reconnaissance synchronique d'autres horizons de crédibilité que celui de chacun.

\section{L'axe Washington-Moscou-New Delhi : "géopolitique heuristique » ou réalité ?}

\section{Retour à la table des matières}

La positivité de la géopolitique incite, et c'est essentiel, à ne pas perdre de vue les dénivellations de la puissance mondiale, les écarts béants qui séparent les capacités stratégiques des acteurs en présence. La thèse très «à la mode», mais pas si nouvelle parce qu'elle se trouvait déjà chez des auteurs comme Silviu Brucan 24 , sur la dissolution de la puissance, n'est pas partagée par les dirigeants des grands États tels que les États-Unis, la Chine ou l'Inde, ni d'ailleurs par ceux d'États plus petits en situation d'insécurité chronique. La divergence qui se remarque entre Européens et Américains quant à la conception de la puissance en général, et à l'usage de la force militaire en particulier, est ici significative de l'influence du contexte et de la « distribution des rôles». Tandis que les premiers sont tout prêts à croire la puissance dépassée, les seconds sont convaincus de sa nécessité pour la défense de la démocratie et pour leur propre sécurité. La décision de l'Administration de George W. Bush d'augmenter sensiblement les dépenses militaires en conformité avec les développements technologiques de la «Révolution dans les affaires militaires» 25 montre bien que les gendarmes du monde ne sont plus les puissances européennes! Car le "grand jeu » des puissances n'est pas prêt de s'arrêter. C'est ainsi que la spectaculaire alliance qu'ont conclue, au printemps 2002, les États-Unis et la Russie donne une idée de la configuration géopolitique du monde qui s'esquisse en ce début de XXIe siècle. Elle semble s'organiser de plus en plus nettement en fonction d'un axe stratégique

24 Silviu Brucan, The Dissolution of Power, New York, Knopf Éd., 1974.

25 Laurent Murawiec, La guerre au XXIe siècle, Paris, Odile Jacob, 2000. 
majeur, l'axe Washington-Moscou-New Delhi. En effet, de part et d'autre de cet axe, se trouvent les deux ensembles géopolitiques (Asie-Pacifique et Asie-Méditerranée) les plus problématiques pour les États-Unis parce qu'ils contiennent chacun un perturbateur potentiel ou actif de l'ordre mondial que gèrent les vainqueurs de la guerre froide. Sachant aussi que, de son côté, la Russie, au sud-est et au sudouest, et que du sien, l'Inde, au nord-est et à l'ouest, sont directement au contact des deux espaces stratégiques signalés, certains rapprochements s'expliquent aisément. Sur le théâtre Asie-Pacifique, le souci de contenir la montée en puissance de la Chine concerne aussi bien les États-Unis, par rapport à Taiwan et à la Corée du Sud notamment, que la Russie, qui s'inquiète pour sa province d'Extrême-Orient, et que l'Inde, toujours méfiante à l'égard de l'autre géant asiatique. L'issue attendue est celle d'un jeu interétatique classique, fait de négociations et de marchandages diplomatiques, militaires et commerciaux, dans le but de canaliser l'expansion de la Chine et de fixer sa position dans le système mondial. Sur le théâtre Asie-Méditerranée, qui va de l'Inde à Gibraltar, en passant par le Moyen-Orient, l'Asie centrale, les Balkans et l'Afrique du Nord, où se concentrent les priorités actuelles des Américains et des Russes, la situation est plus compliquée. D'abord, parce que, sauf la présence de l'«État voyou» irakien, selon la terminologie sécuritaire des États-Unis, le perturbateur est ici le terrorisme islamiste [59] qui se fond au sein des sociétés nationales musulmanes, et auquel il faut livrer une longue et difficile guerre de réseaux. Ensuite, les possibilités de manoeuvre y sont parfois limitées en raison des jeux d'alliances - pas toujours très compatibles - des uns et des autres, tels ceux des États-Unis, qui entendent garantir la sécurité d'Israël, avec plusieurs États musulmans (Arabie Saoudite, Égypte, Afghanistan, Turquie, etc.). Tel aussi celui de la Russie avec l'Iran, lequel n'est pas encore vraiment réconcilié, c'est le moins que I'on puisse dire, avec les États-Unis. Quant à l'Inde, «vieille amie » de Moscou, ses relations avec la Maison-Blanche sont plutôt nerveuses, en particulier à cause du soutien sans faille que les États-Unis apportent au Pakistan depuis les débuts de la guerre froide, à quoi s'ajoute le différend quant au développement de l'arme nucléaire indienne. Cependant, à l'occasion de la dernière crise au Cachemire, la pression exercée par Washington sur Islamabad en faveur d'une désescalade et 
pour que le Pakistan démontre la même fermeté envers les réseaux islamistes cachemiris qu'à l'égard de ceux qui soutiennent Al-Qaïda, devrait permettre une amélioration tangible des relations américanoindiennes.

Certes, l'axe Washington-Moscou-New Delhi est plus heuristique, à savoir susceptible de générer des explications, qu'il n'existe concrètement sur les plans diplomatique et militaire, et ce, même de façon indirecte, en considérant le rôle d'intermédiaire que la Russie est en mesure de jouer. Surtout, son éventualité reste à la merci d'interactions négatives entre les puissances concernées. On peut se demander jusqu'à quel point Moscou est prêt à accepter la présence de troupes américaines en Asie centrale. Pour l'heure, l'accord pétrolier russoaméricain signé le 24 mai 2002, indispensable à la survie financière de la Russie, laisse à penser que le Kremlin est résigné à faire d'importantes concessions, tandis que les Occidentaux savent qu'il leur faudra de plus en plus compter sur le pétrole russe. D'ici dix à vingt ans, il sera nécessaire, pour faire face à la croissance de la demande mondiale, de mettre en place des capacités de production équivalentes, dans un premier temps, au double de la capacité actuelle de l'Arabie Saoudite, puis, dans un second temps, à $130 \%$ du total des capacités actuelles de tous les pays de l'Organisation des pays exportateurs de pétrole (OPEP) réunis 26 . Il s'agit de trouver hors du Moyen Orient, véritable Oil-Heartland mondial puisqu'il renferme les deux tiers des réserves de la planète, des solutions de substitution 27 . Quant à l'Inde, pauvre en la matière et nettement plus isolée qu'autrefois dans un contexte régional marqué par la montée des fondamentalismes et les radicalisations identitaires, elle ne trouverait que des avantages à s'entendre, à la fois, avec la Russie et les États-Unis. En tout état de cause, selon la tradition védique de l'Arthashastra ${ }^{28}$, Moscou et Was-

26 Nicolas Sarkis, « Barils de pétrole et barils de poudre au Proche-Orient», Le Monde diplomatique, juin 2002.

27 Par Oil-Heartland, il faut comprendre la région pétrolifère clé du golfe AraboPersique, qui pourrait se prolonger au nord par le bassin de la mer Caspienne, et dont le contrôle assure celui de l'économie productive mondiale.

28 L'Arthashastra est le grand texte politique de I'Inde ancienne. Il est attribué par la tradition à Kautiliya, ministre brahmane du roi Chandragupta (321-297 av. 
hington figurent bien comme ses deux alliés de revers capables de neutraliser des voisins toujours jugés dangereux et maléfiques. Si tel était le cas, l'Union indienne entrerait dans le « grand jeu » conçu par des membres éminents de l'Administration Bush (Richard Cheney, Condoleezza Rice), qui ont une vision beaucoup moins centrée sur l'Europe que d'autres politologues américains célèbres, de culture plus classique.

[60]

\section{Critique géoéconomique de l'homogénéité du modèle capitaliste mondial}

\section{Retour à la table des matières}

La lutte pour l'hégémonie est la raison précise qui rend pertinent, aux yeux d'Immanuel Wallerstein, l'usage du vocable « géopolitique ». En effet, selon l'universitaire américain, cette lutte qui a opposé à trois reprises dans l'histoire du système mondial moderne, entre 1793 et 1945, une puissance continentale (France ou Allemagne) à une puissance maritime toujours victorieuse (Angleterre puis États-Unis) grâce à l'aide d'alliés continentaux, forme l'arrière-plan géopolitique de l'expansion de l'économie mondiale capitaliste ${ }^{29}$. Le succès de cette dernière apparaît définitif avec la fin du léninisme qui a marqué une rupture fondamentale dans la géoculture de l'économie capitaliste mondiale.

C'est qu'au sein même de cette géoculture, appelée ainsi «par analogie avec la géopolitique, non pas parce qu'elle est supralocale ou supranationale, mais parce qu'elle représente l'arrière-plan culturel dans

J.-C.). Dans le livre VI est exposée la théorie des grands cercles par rapport auxquels l'Inde n'a cessé de se situer en fonction d'un principe simple (le voisin est l'ennemi, le voisin du voisin est l'ami) transposable du niveau régional au niveau planétaire. Voir Jean-Alphonse Bernard, L'Inde. Le pouvoir et la puissance, Paris, Fayard, 1985, notamment p. 16-17, et chap. 9.

29 Immanuel Wallerstein, Geopolitics and Geoculture. Essays on the Changing World System, Cambridge, Cambridge University Press, réédition 2001. 
lequel opère le système mondial $\gg 30$, existent différentes forces qui rejettent les présentations universalistes du libéralisme. Autrement dit, si la mondialisation capitaliste est un fait acquis, géopolitique et géoculture sont des notions parfaitement justifiées pour signifier, d'abord, l'analyse des luttes pour la puissance et pour la richesse qui la sous-tendent, et, ensuite, l'étude du rapport de la mondialisation aux identités ou aux différences culturelles qui s'affirment et surgissent de toutes parts. Les deux catégories insistent en effet sur les divergences d'intérêts et sur les revendications différentialistes dans la mondialisation.

\section{L'hétérogénéité spatiale et temporelle de l'économie mondiale}

\section{Retour à la table des matières}

Wallerstein, pour sa part, distingue plusieurs types de rejets: la pensée de 1968, le racisme, le sexisme ou encore ce qu'il dénomme « la nouvelle science» parce qu'elle s'en prend à la tradition baconienne/newtonienne, et qui, à la façon dont il la décrit, correspond au postmodernisme le plus radical. Toutefois, et I. Wallerstein l'explique depuis ses premiers livres sur le capitalisme mondial, les résistances les plus marquées proviennent des nationalismes d'essence économique et sociale. Car, bien entendu, le temps, c'est-à-dire les cycles d'évolution des différentes sociétés intégrées, n'est pas le même dans tout l'espace de l'économie mondiale.

Aujourd'hui, on observe que l'extension à toute la planète de la division du travail capitaliste, couplée avec l'inflation démographique du monde, engendre une offre de travail considérable mais inégalement répartie, qui tire partout, et y compris dans les pays développés, la rémunération du travail vers le bas. Dans le capitalisme mondial, les déséquilibres de la démographie économique entraînent, dès lors, la réactualisation des contradictions qui ont caractérisé dans le passé, au sein des économies nationales, les rapports entre le capital et le tra-

30 Ibid. 
vail. De plus en plus va se poser la question de la compatibilité d'un commerce ouvert, réputé faire le bonheur de tous, avec la présence dans son aire des « pays à bas salaires et à capacités technologiques » que pointe Pierre-Noël Giraud 31 : comment l'Organisation mondiale du commerce $(O M C)$ réussira-t-elle s'adapter à 600 millions de paysans chinois? Pour peu que la revendication sociale, inhérente à l'insécurité économique et à la dépréciation du prix du travail, se conjugue avec un raidissement identitaire, alors le risque de rébellion dans les [61] pays développés, déjà signalé par Barry Buzan 32 , devrait encore se renforcer. Par conséquent, si l'on entend caractériser les hétérogénéités de l'espace économique mondial et mettre en relief les éléments qui, au sein de l'économie-monde, engendrent les polarisations externe (Nord/Sud) ou interne (à l'intérieur de tous les États, riches et pauvres), on est fondé à approcher ces phénomènes en termes géoéconomiques.

La géoéconomie n'est rien moins que la géopolitique des forces économiques, des tensions qui accompagnent la dernière forme-étape du capitalisme (la mondialisation) dans laquelle les États, à l'exception des plus puissants, ont perdu l'essentiel de leurs marges de manoeuvre, sans que d'autres instances de régulation aient vraiment pris le relais, ce qui rend plausible une autre et nouvelle fragmentation de l'espace économique mondial. On peut dès lors considérer, avec Nicolas BardosFéltoronyi, que la géo-économie se nourrit de l'étude des rapports économiques alternativement conflictuels et coopératifs, et de celle du « développement inégal tant spatial et temporaire que sectoriel » 33.

On peut la concevoir également comme l'approche spatiale de cette discipline particulière de la science politique, qui s'est beaucoup développée ces dernières années dans les pays anglo-saxons, à savoir l'économie politique internationale..., à condition de lui ajouter ce qui

31 Pierre-Noël Giraud, L'inégalité du monde. Économie du monde contemporain, Paris, Gallimard, 1996.

32 Barry Buzan, People, States and Fear. An Agenda for International Security Studies in the Post-Cold War Era, Boulder (Co.), Lynne Rienner Publishers, $2 e$ éd., 1991.

33 Nicolas Bardos-Féltoronyi, Géoéconomie. État, espace, capital, Bruxelles, De Boeck, 1991. 
lui manque! En effet, si l'objet de cette dernière est l'interaction du politique et de l'économie au niveau planétaire, elle tend à concentrer ses travaux - en raison du succès du capitalisme et de la transparence retrouvée du monde - sur la maximisation individuelle de l'utilité et sur les mécanismes de l'échange, laissant ainsi de côté les phénomènes de pouvoir et de manipulation ou encore les risques d'une crise systémique. En l'occurrence, il pourrait s'agir d'un retour « de l'économie de la dépression» provoquée par la suraccumulation du capital au niveau mondial, d'un côté, et par la sous-consommation des plus nombreux, de l'autre, comme s'en est inquiété Paul Krugman ${ }^{34}$. En Europe, en particulier, le vieillissement de la population, outre les charges à venir qu'il suppose et qui commencent seulement à préoccuper les gouvernants, sera un facteur d'anémie aggravée en raison de la saturation de la demande et de la réduction des occasions d'investir, alors même que le niveau des investissements est déjà faible.

\section{Une régulation par zones de l'économie mondiale?}

\section{Retour à la table des matières}

Les études sur la globalisation n'ont que trop tendance à éluder les disparités et les déséquilibres de l'espace économique planétaire qui rendent aléatoire ou même illusoire l'efficacité de la régulation mondiale. En effet, dans le prolongement de ce qui existe déjà, il ne commencera à en être autrement que «lorsque les normes de l'Organisation internationale du travail (OIT) s'imposeront dans les négociations commerciales, lorsque la Banque mondiale coordonnera son action avec le Fonds monétaire international (FMI), lorsque l'Organisation des Nations unies pour l'éducation, la science et la culture (Unesco) sera capable de donner un contenu à la diversité culturelle, lorsqu'une organisation internationale soutiendra les enjeux environnementaux, [et lorsque] l'OMC [...] n'arbitrera plus les conflits sur

34 Paul Krugman, Pourquoi les crises reviennent toujours?, Paris, Le Seuil, 2000. 
les seules bases de l'universel marchand $» 35$. Or il ne s'agit là que des dispositions minimales pour un horizon incertain.

[62]

En attendant, le creusement des inégalités, la démarcation des priorités économiques et des logiques sociales suggéreraient plutôt une régulation par zones, la plus à même d'assumer la différenciation régionale de l'économie globale. Entre l'utopique régulation de l'économie-monde et le dépassement structurel et fonctionnel des États, des solutions existent, telles que la reterritorialisation monétaire que l'Europe semble en passe de réussir. Celle-ci est d'autant plus la bienvenue qu'avec la globalisation de la finance et de ses dérèglements spéculatifs les crises financières tendent à se répéter. Certes, il faut compter avec l'épreuve du temps, et l'euro ne saurait se suffire à lui-même pour garantir la croissance de l'économie européenne dans la stabilité monétaire, mais il est une preuve tangible de la pertinence de l'option régionale plutôt que mondiale.

On devrait aussi s'interroger davantage sur le caractère sélectif des aides accordées par le FMI. L'interaction avec les intérêts politiques de son principal bailleur de fonds n'y est pas pour rien. Il suffit, pour en avoir une bonne illustration, de comparer la faiblesse de l'aide accordée à la Russie en 1998 au soutien systématique dont bénéficie la Turquie, et au quasi-abandon de l'Argentine. La notion de mondialisation est à la fois trop euphémique et trop édulcorante des rivalités du champ économique.

Il se trouve aussi que la logique sécuritaire, en matière énergétique, alimentaire ou encore informationnelle, se satisfait mal de la logique du marché. Le pétrole, dont l'ère devrait toucher à sa fin au milieu du XXIe siècle, en constitue toujours, comme on l'a vu, l'une des meilleures démonstrations. Le marché des biens et des services est luimême livré aux rapports de force, car, pour maintenir ou augmenter leurs valeurs actionnariales respectives, les entreprises se concen-

35 Élie Cohen, L'ordre économique mondial. Essai sur les autorités de régulation, Paris, Fayard, 2001. 
trent dans des oligopoles de façon à garantir ou à étendre leur part de marché 36 .

Toutes les formes de polarisation que l'on constate au coeur même de la mondialisation renvoient à la question de l'ordre et du désordre. En démultipliant le nombre des acteurs et les espaces d'action, n'ajoute-t-elle pas à l'anarchie internationale? Parce que son objet d'étude est l'organisation des forces économiques dans l'espace, la géoéconomie est en mesure de répondre, contextuellement, à ces interrogations.

\section{Perspectives géoculturelles}

Retour à la table des matières

Le terme " géoculture», dans le sens que lui donne I. Wallerstein, a pour fonction de faire ressortir toute la diversité de l'arrière-plan culturel de la mondialisation. Il voit même en lui le « champ de bataille idéologique du système-monde moderne » 37 , ce qui lui a été reproché au titre qu'il privilégiait l'une des deux acceptions de la culture, celle qui divise aux dépens de celle qui réunit ${ }^{38}$. D'autres auteurs lui ont donc opposé le concept de culture globale, avancé avec précaution, mais trop vite réifié par le langage journalistique. Car la globalisation de la culture, si elle est rendue irrésistible par l'explosion de la communication universelle, n'équivaut pas à son homogénéisation. Au contraire, selon un éminent représentant du courant anthropologique des cultural studies, A. Appadurai, la rencontre des migrations de masse et des médias électroniques sans frontières permettrait la reconstruction des identités à une plus grande échelle, en maintenant le

36 Immanuel Wallerstein, « Culture as the Ideological Battleground of the Modern World-System », in Mike Featherstone, Global Culture: Nationalism, Globalization and Modernity. A Theory, Culture and Society Special Issue, Londres, Sage, 1990.

37 Ibid.

38 Roy Bone, « Culture and the World-System », in M. Featherstone, op. cit., p. 5765. 
lien culturel avec l'aire d'origine ${ }^{39}$. Dans cette perspective, l'objet central de la géoculture va consister à problématiser la mise en place d'un communautarisme au niveau de la planète.

[63]

\section{Les cultures sont-elles solubles dans la mondialisation?}

\section{Retour à la table des matières}

La théorie de la modernisation, telle qu'elle a été longtemps partagée par les libéraux comme par les marxistes, postule que la capacité adaptative de l'humanité, au terme d'étapes définies, conduirait à la création d'une vaste communauté humaine, solidaire et coopérative, dans laquelle se dissoudraient les frontières et les différences. Selon $R$. Robertson, et d'autres sociologues avec lui, la globalisation va dans ce sens. Les États-nations ne constituent déjà plus un monde en interaction mais une unité ("the concrete structuration of the world as a whole ") 40 , un ensemble unique au sein duquel le processus d'intégration a pris son autonomie, principalement en raison de l'expansion et de l'intensification des flux culturels globaux.

Une culture globale est en train de naître, qui pose néanmoins le problème de sa vraie nature. Car, pour être réellement globale, elle devrait être la parfaite symbiose des cultures particulières. Mais jusqu'au XXe siècle tout au moins, la convergence a cédé le pas à l'occidentalisation. En effet, si l'on considère une à une les catégories de la modernité proposées par Jürgen Habermas 41 , les Occidentaux,

39 A. Appadurai, op. cit.

40 Roland Robertson, « Mapping the Global Condition: Globalization as the Central Concept », in M. Featherstone, op. cit.

41 « Le concept de modernisation désigne [...] la capitalisation et la mobilisation des ressources, le développement des forces productives et l'augmentation de la productivité du travail ; il désigne également la mise en place de pouvoirs politiques centralisés et la formation d'identités nationales; il désigne encore la propagation des droits à la participation politique, des formes de vie urbaine et de 
et particulièrement les Européens, ont tout inventé. Ce qui représente probablement une limite à la globalisation, en raison précisément de la sénescence de l'Occident, et du fait qu'il est de plus en plus minoritaire sur le plan démographique. Mais si la culture globale n'est pas la conséquence de l'expansion mondiale de la modernité occidentale, que peut-elle être? Un autre hégémonisme culturel, religieux par exemple? Ou bien une "hybridation», comme le pense Mike Featherstone qui la perçoit comme formée, d'une part, de sous-ensembles culturels étrangers les uns aux autres, précipités les uns contre les autres, et, d'autre part, de tierces cultures, c'est-à-dire d'éléments culturels sans identité se transmettant d'un lieu à un autre 42 ? Pour R. Robertson, une telle composition procède de la rencontre des flux globaux et des identités locales, d'où son penchant pour le néologisme glocalization afin de distinguer ce processus 43 . Ce dernier ne saurait être assimilé à une fusion, prévient-il néanmoins. Si la culture globale résulte de l'interconnexion grandissante des cultures locales, réduites ou vastes, il est exclu de voir en elle l'homogénéisation de toutes les cultures ${ }^{44}$.

\section{Renouvellement mimétique de paradigmes anciens}

\section{Retour à la table des matières}

Or il y a dans cette réserve un argument qui peut suffire à rendre, aux yeux d'un grand nombre d'observateurs, cette notion inopportune. C'est l'avis d'Anthony D. Smith 45. Ce sociologue britannique, bien qu'il rejette le primordialisme qui prétend que les identités sont des données naturelles, conclut à l'existence et à la pérennité des ethnies, au prix du paradoxe suivant : la mutabilité de l'ethnie dans la persistance

l'instruction publique: il désigne enfin la laicisation des valeurs et des normes, etc. », in Jürgen Habermas, Le discours philosophique de la modernité, Paris, Gallimard, 1988.

42 M. Featherstone, op. cit., p. 1-14.

43 R. Robertson, « Mapping the Global Condition... », op. cit.

44 Ibid.

45 Anthony D. Smith, « Towards a Global Culture?», in M. Featherstone, op. cit. 
et [64] sa persistance dans le changement 46 . Il déclare ne pas être surpris par le renouveau des ethnonationalismes dans les sociétés industrielles. Il y voit une nouvelle phase de mobilisation d'identités démotiques (c'est-à-dire d'essence majoritaire populaire, et non pas « construite» par une élite) qui remontent au XVIIIe siècle, et même au-delà, et dont ce qu'il appelle les mythomoteurs respectifs restent les référents fondamentaux 47 . A. D. Smith désigne par là les complexes particuliers de symboles et de valeurs partagés, de styles et de genres de vie, qui ont pu changer en fonction des contextes historiques, des contacts et des stratégies d'acteurs, lesquels acteurs, à un moment donné, définissent les contours de ces identités.

Fidélité aux origines et renouvellement par mimétisme sont aussi, selon A. Appadurai, au coeur de la problématique contemporaine du rapport global/local rendu plus complexe par la transnationalité des cultures et par la reconstruction des identités au sein des diasporas. Parce que «les groupes migrent, se rassemblent dans des lieux nouveaux, reconstruisent leur histoire et reconfigurent leur projet ethnique 48 , la communautarisation du monde (l'auteur parle d'une « fédération de diasporas ») semble plus proche de la réalité que l'avènement de la culture globale, pleine et entière. Cet anthropologue constate la mise en échec de la modernité par la conjonction des médias et des migrations, qui entraîne la multiplication et la juxtaposition, dans toutes les sociétés multiculturelles, des «lieux postnationaux» 49. A. Appadurai entend, par là, la prolifération de micro-espaces diasporiques clos, "enfermés dans leur petite bulle», mais connectés entre semblables. Il y voit aussi l'origine d'une ethnicité moderne qui dépasse la perspective primordialiste, sachant qu'au substrat conventionnel des sentiments ethniques et historiques se substituent « des produits complexes de l'imagination locale »50. Tout autant que l'ancienne, cette ethnicité moderne est porteuse d'une dynamique d'implosion et

46 Anthony D. Smith, The Ethnic Origins of Nations, Londres, Blackwell Publishers, 1999.

47 Ibid.

48 A. Appdurai, op. cit.

49 Ibid.

50 Ibid. 
d'explosion. Elle pose les questions de la coexistence des groupes humains et du devenir des États nationaux quand, au coeur de leurs populations hétérogénéisées, s'exprime la volonté des uns et des autres de conserver les différences.

C'est à la lumière de cette tendance générale qu'il convient sans doute d'analyser la progression, ces dernières années, des mouvements dits «nationaux-populistes», à l'occasion des différents scrutins organisés en Europe. Ainsi, en France, la présence du candidat du Front national au second tour de l'élection présidentielle peut se comprendre - en suivant les explications de Pierre-André Taguieff, bien qu'il n'utilise pas explicitement le terme - comme l'expression du premier grand vote communautaire. Là où les analyses électorales classiques ne fonctionnent plus, le sociologue en retrouve une dans la crise de la modernité 51 . Il observe l'émergence de trois données fondamentales qui éclairent la surprise du 21 avril 2002, parce qu'elles ont influencé directement l'électorat concerné : «L'effacement de l'homogénéité ethnique et culturelle des populations nationales », sous l'action d'une immigration clandestine de plus en plus massive, qui « rend hautement problématique le statut des citoyens d'un Étatnation »; le rejet de la mondialisation par toute une catégorie de « sans-espoirs », en particulier dans les régions où s'étendent les friches industrielles; l'affaiblissement de l'État au nom d'une «Europe » qui n'apporte pas de solution de substitution dans la mesure où la seule préoccupation de Bruxelles est perçue comme la volonté de soumettre toutes les sociétés européennes aux règles du marché mondial. Ces causes [65] n'étant pas prêtes de disparaître (du fait qu'elles relèvent de tendances géopolitiques lourdes, et parce qu'elles ont peu à voir avec la résurgence d'une idéologie du passé, sauf pour une infime minorité), il faut s'attendre à l'installation durable de tels comportements électoraux aux ressorts avant tout culturels.

51 Pierre-André Taguieff, «Sortir d'un antifacisme de parade», Le Figaro, 25-26 mai 2002. 


\section{Le « nouveau multilatéralisme »: un dialogue intercivilisationnel}

\section{Retour à la table des matières}

Au sein de la communauté des spécialistes en relations internationales, quelques-uns ont pris acte de la dimension culturelle et civilisationnelle de la matière - avec ses aspects parfois irréductibles -, tout en récusant la thèse de Samuel $P$. Huntington (ils lui ont néanmoins reconnu le mérite d'avoir réintroduit un paramètre, négligé depuis longtemps bien qu'essentiel, de la vie internationale).

R.W. Cox et Stephen Gill, puisque c'est d'eux qu'il s'agit principalement, font de la reconnaissance de la diversité culturelle et de l'hétérogénéité des approches civilisationnelles, la base d'un « nouveau multilatéralisme» 52 . Ils le conçoivent dégagé des considérations strictement marchandes, respectueux des différents horizons et, par conséquent, des différentes images du monde produites à partir de ceux-ci. Selon eux, le succès de la gouvernance mondiale est tributaire de la connaissance et du respect des traditions autant que des changements qui opèrent au sein d'une géoculture nécessairement pluraliste dans ses idées sur la nature du monde réel, dans ses valeurs, dans ses sens de la vie. Le nouveau multilatéralisme étant pensé comme l'alternative à la globalisation hégémonique telle qu'on la connaît, l'intersubjectivité constitue la seule base réaliste (c'est pourquoi R.W. Cox a intitulé l'un de ses derniers livres The New Realism) d'une coexistence et d'un enrichissement mutuels entre des civilisations qui ont pu, un jour, entrer en conflit. Les deux politologues américains se situent dans une démarche postwestphalienne ou postnationale, commune à beaucoup d'autres aujourd'hui.

Cependant, il n'est pas certain que la nouvelle puissance des flux globaux et des réseaux diasporiques doit être systématiquement opposée à celle des États-nations. Il est, en effet, un certain nombre de

52 Robert W. Cox, The New Realism. Perspectives on Multilateralism and World Order, New York, Macmillan Press, 1997, introd., p. XV-XXX. 
cas, comme celui de la Chine, où, quand l'espace originel reste homogène et entretient un projet national, l'extension de la diaspora et la multiplication des réseaux au-delà du territoire de référence articulent avec ce dernier un macrosystème puissant. Or, dans la lutte pour l'hégémonie qu'est, au fond, la globalisation culturelle, et en l'absence de toute universalisation cohérente, de tels macrosystèmes auront nécessairement leur mot à dire.

\section{Géopolitique/géostratégie: couple référentiel d'une même analyse spatio-politique}

\section{Retour à la table des matières}

La globalisation économique et culturelle, il faut en convenir, n'est pas synonyme de pacification du monde. Elle politise les différences, et elle exacerbe tous les rapports en faisant de la concurrence son principe cardinal. Du même coup, comme l'avait bien pressenti JeanPaul Charnay, la stratégie est devenue à la fois " globale » et « différentielle $\gg 53$. Elle est d'abord globale parce qu'elle concerne tous les acteurs, à l'échelle planétaire. Elle est ensuite différentielle parce qu'elle se dissocie en fonction des champs dans lesquels elle se déploie. Devenue multidimensionnelle et protéiforme, elle a perdu sa stricte pertinence militaire pour en acquérir une nouvelle [66] d'ordre systémique, mais au prix, il est vrai, d'un certain affadissement de la notion, ce que son épistémologue constate de la façon suivante: «La stratégie, poursuivant ses transformations sémantiques, tend à prendre un sens encore plus étendu par l'évacuation de l'idée même d'antagonisme [...] et donc ne signifie plus usage d'une série de moyens déterminés, mais adaptations générales aux fins poursuivies quels qu'en soient les moyens. » 54 Par voie de conséquence, la géostratégie s'élargit également. Elle devient, à l'occasion de cette mutation considérable, la com-

53 Jean-Paul Charnay, Essai général de stratégie, Paris, Champ libre, 1973.

54 Ibid. 
préhension et l'adaptation des comportements stratégiques dans l'espace de chaque acteur, quel qu'il soit, État, firme, individu, groupe d'intérêts, etc. C'est la raison pour laquelle nous avons proposé de la distinguer de la géopolitique en fonction de leurs caractérisations propres, conceptuelle pour celle-ci, instrumentale pour l'autre. À savoir que la géopolitique serait l'étude de l'étant de l'espace mondial, de sa configuration et de ses dynamiques, en même temps que celle des positions respectives des acteurs dans le système mondial. Tandis que la géostratégie serait celle de l'actant, puisqu'elle consisterait à analyser les actions stratégiques, dans les différents champs que contient la configuration.

La stratégie façonnant autant qu'elle détruit, l'acteur s'évertue à modifier le contexte dans lequel il se meut. Gary Goertz, qui est l'un des rares politologues à avoir analysé ce concept avec précision, en distingue trois catégories qui agissent, en particulier, sur le comportement des États: la structure géographique de la puissance internationale, le contexte historique qui montre pourquoi et comment les actions passées sont reliées aux comportements présents, et l'environnement normatif fait de valeurs, de règles et de normes juridiques 55 . Chacune d'entre elles est tantôt un obstacle, tantôt une opportunité. La géostratégie en soi consiste alors à gérer dans l'espace, en fonction de son immense diversité, et de plus en plus au niveau global, ses ressources et ses contraintes, ce que les entreprises multinationales devenues transnationales savent faire depuis longtemps. On le comprend facilement, l'aspect déterminant de la variable spatiale dans la stratégie multidimensionnelle des acteurs légitime la référence à la géostratégie.

La confrontation désormais mondiale des intérêts, des valeurs, des symboles, des visions de l'Histoire et du devenir, que provoque la globalisation, entraîne une prise en considération de l'espace de vie dans toutes ses dimensions et avec toutes ses hétérogénéités. Surtout que celles-ci mettent régulièrement en échec les processus de régulation issus d'une conception trop uniforme et trop préconçue du monde. La multiplication du préfixe "géo » devant une série de substantifs, qui

55 Gary Goertz, Contexts of International Politics, Cambridge, Cambridge University Press, 1994. 
renvoient chacun à un champ d'activité, révèle simplement la nécessité, de plus en plus ressentie, d'intégrer toute la différenciation régionale et locale du mouvement d'unification du monde. Aussi, afin d'éviter l'inflation dudit préfixe, il serait préférable d'admettre, une fois pour toutes, que la géopolitique est systémique et, par conséquent, qu'elle englobe tous les champs concernés et interactifs 56 .

Fin du texte

56 G. Dussouy, Quelle géopolitique au XXIe siècle?, op. cit. 